\title{
TARPSY 1.0: Das neue Tarifsystem für die stationäre Psychiatrie
}

\section{Beatrix Meyera ${ }^{a}$ Barbara Rohnerb}

${ }^{a}$ Leiterin Abteilung Stationäre Versorgung und Tarife; ${ }^{b}$ Wissenschaftliche Mitarbeiterin Abteilung Stationäre Versorgung und Tarife

Die Tarifstruktur TARPSY Version 1.0 basiert auf leistungsbezogenen Tagespauschalen. Auf das ursprünglich vorgesehene Fallpauschalenelement wird richtigerweise verzichtet. Die verbindliche Einführung von TARPSY ist für die Erwachsenenpsychiatrie per 1.1.2018 und für die Kinder- und Jugendpsychiatrie per 1.1.2019 geplant.

Leistungen der stationären Psychiatrie sollen schweizweit mit einem einheitlichen leistungsbezogenen Tarifsystem vergütet werden - so will es das Gesetz. Die SwissDRG AG unterbreitet die Tarifstruktur TARPSY Version 1.0 demnächst dem Bundesrat zur Genehmigung. Kalkuliert ist TARPSY Version 1.0 anhand der Spitaldaten der Jahre 2014 und 2015. Die FMH hat sie zusammen mit den betroffenen Fachgesellschaften analysiert und dazu Stellung genommen.*

\section{Leistungsbezogene degressive Tages- pauschalen}

Das Tarifsystem TARPSY Version 1.0 basiert auf leistungsbezogenen Tagespauschalen. Das ursprünglich vorgesehene Fallpauschalenelement ist für die Psychiatrie ungeeignet und fällt nun konsequenterweise

\section{Der Verzicht auf das Fallpauschalenelement} ist folgerichtig.

weg. Die Patientenaufenthalte bzw. Fälle werden anhand der Hauptdiagnose der Patienten in zehn möglichst kostenhomogene psychiatrische Basis-Kostengruppen (Basis-PCGs) unterteilt. Für mehrere dieser Basis-PCGs erfolgt eine zusätzliche Differenzierung nach folgenden Kriterien: Nebendiagnose, Symptomintensität sowie Alter. Eine Basis-PCG ist in maximal drei PCGs unterteilt. Dadurch ergeben sich insgesamt 22 psychiatrische Kostengruppen.

* Zur ausführlichen Stellungnahme der FMH zu TARPSY Version $1.0 \mathrm{vgl}$. www.fmh.ch $\rightarrow$ Stationäre Tarife $\rightarrow$ Positionen

$\rightarrow$ Stellungnahmen.
Je nach PCG und je nach Aufenthaltsdauer eines Patienten weist der Fall ein unterschiedliches Tageskostengewicht auf. Dieses ist innerhalb einer PCG bei kürzeren Aufenthalten höher (oder zumindest gleich hoch) als bei längeren Aufenthalten. Die Aufenthalts- dauer bemisst sich aus Pflegetagen inklusive des ganzen Entlassungs- bzw. Verlegungstags. Das Tageskostengewicht multipliziert mit der Aufenthaltsdauer und dem von den Versicherern und Spitälern verhandelten Basispreis ergibt den Erlös eines Falls.

\section{Symptomintensität und Nebendiagnosen als sich ergänzende Kriterien}

Die Symptomintensität misst man in der Erwachsenenpsychiatrie mit folgenden drei HoNOS-Dimensionen [1]: hyperaktives aggressives Verhalten, absichtliche Selbstverletzung sowie körperliche Erkrankung/ Behinderung. Die SwissDRG AG stellte mit ihren Datenanalysen fest, dass Patienten mit einer hohen Symptomintensität oder mit bestimmten Nebendiagnosen teilweise einen erhöhten Behandlungsaufwand verursachen [2]. Entsprechend können diese Fälle in eine besser bewertete PCG gelangen. Dabei ist es ausreichend, wenn der Patient entweder eine hohe Symptomintensität oder alternativ mindestens eine der relevanten Nebendiagnosen aufweist. Die SwissDRG AG listet in ihrem Definitionshandbuch für die betroffenen PCGs auf, welche HoNOS-Dimensionen bzw. Nebendiagnosen relevant sind.

Die vorliegenden Nebendiagnosenlisten entsprechen jedoch teilweise nicht den klinischen Erfahrungen. Dies dürfte auf die ungenügende Datenqualität der erfassten Nebendiagnosen zurückzuführen sein [3]. Deshalb gilt es, die Datenqualität zu verbessern und die Nebendiagnosenlisten künftig auf einer validen Datengrundlage zu erstellen. Datengestützt soll aus Sicht der FMH für TARPSY Version 2.0 zudem geprüft werden, inwieweit Patienten mit multiplen Nebendiagnosen behandlungsintensiver sind und ob für diese 
Beispiel:

Ein erwachsener Patient mit Hauptdiagnose «Schizophrenie» und komplizierenden somatischen Nebendiagnosen

wird 18 Tage stationär behandelt:

- Psychiatrische Basis-Kostengruppe (Basis-PCG): TP25

- Psychiatrische Kostengruppe (PCG): TP25B

- Kostengewicht pro Tag: 1.067

- Basispreis: Verhandelt zwischen Versicherer und Spitäler

Erlös $=$ Kostengewicht pro Tag $\mathrm{x}$ Aufenthaltsdauer in Tagen $\mathrm{x}$ Basispreis in CHF

$\begin{array}{llll}= & 1.067 & \times & \end{array} 8$ Basispreis in $\mathrm{CHF}$

\section{TP25 Schizophrene oder akut psychotische Störungen}

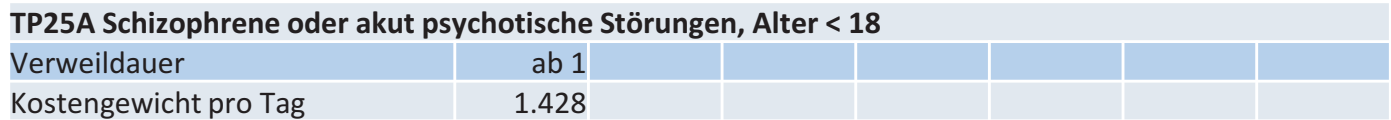

TP25B Schizophrene oder akut psychotische Störungen, Alter > 17 Jahre mit komplizierender somatischer Nebendiagnose

\begin{tabular}{|l|r|r|r|r|r|r|r|}
\hline Verweildauer & 1 & 2 & 3 & 4 & 5 & 6 & 7 \\
\hline Kostengewicht pro Tag & 1.361 & 1.325 & 1.289 & 1.253 & 1.216 & 1.180 & 1.144 \\
\hline Verweildauer & 8 & 9 & 10 & 11 & 12 & 13 & 14 \\
\hline Kostengewicht pro Tag & 1.137 & 1.130 & 1.123 & 1.116 & 1.109 & 1.102 & 1.095 \\
\hline Verweildauer & 15 & 16 & 17 & 18 & 19 & 20 & 21 \\
\hline Kostengewicht pro Tag & 1.088 & 1.081 & 1.074 & 1.067 & 1.060 & 1.053 & 1.046 \\
\hline Verweildauer & 22 & 23 & 24 & 25 & 26 & ab 27 & \\
\hline Kostengewicht pro Tag & 1.039 & 1.032 & 1.025 & 1.018 & 1.011 & 1.005 & \\
\hline
\end{tabular}

TP25C Schizophrene oder akut psychotische Störungen, Alter > 17, ohne komplizierende somatische Nebendiagnose

Details vgI. PCG-Katalog TARPSY 1.0

Quelle: SwissDRG AG, Auszug aus dem PCG-Katalog TARPSY Version 1.0

Abbildung 1: Beispiel für die Berechnung des Erlöses unter TARPSY Version 1.0.

Fälle ein höheres Kostengewicht erforderlich ist. Damit liesse sich eine verbesserte Abbildung von multimorbiden Patienten erzielen, die insbesondere in der Alterspsychiatrie und in den Suchtkliniken keine Seltenheit sind.

\section{Zusätzliche Alterskategorien prüfen}

Die SwissDRG AG bildete für TARPSY Version 1.0 die folgenden drei Alterskategorien: Minderjährige bis 17 Jahre, 18- bis 75-Jährige sowie die Alterskategorie $>75$. Für die Alterskategorie der Minderjährigen bestehen acht PCGs und für die Alterskategorie $>75$ nur eine einzige höherbewertete PCG. Allerdings sind die meisten dieser höherbewerteten PCGs auch für Fälle der übrigen Alterskategorien vorgesehen, sofern die Patienten eine komplizierende Nebendiagnose aufweisen. Beispielsweise werden sowohl Fälle von Patienten der Alterskategorie $>75$ als auch solche der übrigen Alterskategorien mit komplizierender Nebendiagnose in die PCG TP21A eingeteilt (Verhal- tensstörungen bei Missbrauch von Drogen und anderen Substanzen mit komplizierender Nebendiagnose oder Alter $>75$ Jahre).

Aufgrund der mangelnden Datenlage konnte die SwissDRG AG keine Unterscheidung in die Alterskategorien <14 sowie 14-17 vornehmen. Im Gegensatz zur Vorgängerversion TARPSY 0.3 fallen nun die Alterskategorien 65-79 sowie 80+ weg. Gemäss klinischen Erfahrungen bedürfen 65- bis 79-Jährige und speziell über 80-Jährige allerdings einer aufwendigeren Behandlung als jün-

Die Therapie von Minderjährigen und Senioren muss sich künftig leistungsgerecht abbilden lassen.

gere Patienten. Dies auch dann, wenn keine behandlungsintensiven Nebendiagnosen vorliegen. Aus Sicht der FMH ist es deshalb unerlässlich, dass die SwissDRG AG für die TARPSY Version 2.0 eine Einteilung der Altersstufen <14, 14-17, 18-64, 65-79 und 80+ prüft basierend auf einer verbesserten Datengrundlage. 


\section{Psychiatrische CHOP-Kodes erst für spätere TARPSY-Versionen}

Mit der Prozedurenklassifikation CHOP können bisher vor allem Untersuchungen und Behandlungen von Patienten der Akutsomatik statistisch erfasst werden. Die psychiatrischen Prozeduren sind jedoch noch ungenügend in der CHOP abgebildet. TARPSY Version 1.0 berücksichtigt sie deshalb plausiblerweise noch nicht. Zuerst geht es darum, die CHOP bzgl. der psychiatrischen Prozeduren zu verbessern. Hier engagiert sich die «FMH-Arbeitsgruppe CHOP-Revision Psychiatrie» und hat bereits verschiedene Anträge beim Bundesamt für Statistik eingereicht. Bei der Integration der psychiatrischen CHOP-Kodes in spätere TARPSY-Versionen sind der Zusatznutzen für das Tarifsystem und der entsprechende zusätzliche administrative Aufwand sorgfältig abzuwägen. Teure somatische Behandlungen während des psychiatrischen Aufenthalts (Dialyse, teure Medikamente z.B. für onkologische Behandlungen usw.) werden bereits ab TARPSY Version 1.0 anhand des Zusatzentgelte-Katalogs aus der Akutsomatik vergütet [4]. Bei diesen Zusatzentgelten handelt es sich nicht um ein "zusätzliches» Entgelt, sondern um eine separate Vergütung ausserhalb der Tagespauschalen in der Psychiatrie.

\section{TARPSY für Kinder- und Jugendpsych- iatrie vorerst freiwillig}

Die Datengrundlage von TARPSY Version 1.0 ist für die Kinder- und Jugendpsychiatrie ungenügend: Es lagen nur 258 plausible Fälle für <14-Jährige und 1349 plausible Fälle für die 14- bis 17-Jährigen vor. Hinzu kommt, dass in vielen Kantonen Jugendliche aufgrund von mangelnden Jugendpsychiatrie-Plätzen fälschlicherweise in der (kostengünstigeren) Erwachsenenpsychiatrie behandelt werden. Dadurch sinkt das durchschnittliche Kostengewicht für die Behandlung von Minderjährigen und kann den Fehlanreiz setzen, solche Falschplatzierungen verstärkt vorzunehmen.

Angesichts der bestehenden Mängel hatte die FMH beim Verwaltungsrat der SwissDRG AG beantragt, die verbindliche Einführung von TARPSY für die Kinderund Jugendpsychiatrie zu verschieben. Es ist richtig, dass die einzelnen Kliniken nun selbst entscheiden dürfen, ob sie TARPSY Version 1.0 für die Kinder- und
Jugendpsychiatrie per 1.1.2018 einführen oder nicht. Die obligatorische Einführung von TARPSY ist für die Kinder- und Jugendpsychiatrie frühestens per 1.1.2019 vorgesehen. Dieser Zeitplan ist nur dann realistisch, wenn die SwissDRG AG vorab verschiedene Massnahmen einleitet. Beispielsweise ist für TARPSY Version 2.0 die Verwendung des multiaxialen Klassifikationssystems (MAS) zu prüfen. Denn dazu existieren bereits interessante erste Auswertungen für die Vorgängerversion TARPSY 0.3 [5].

\section{Fazit und Ausblick für zukünftige TARPSY-Versionen}

Die FMH bewertet es positiv, dass mit TARPSY Version 1.0 ein auf Tagespauschalen basierendes leistungsbezogenes Tarifsystem vorliegt. Jedoch ist insbesondere die Behandlung von Patienten der Kinder- und Jugendpsychiatrie, der Alterspsychiatrie sowie der Suchtkliniken noch ungenügend abgebildet. Dies hängt u.a. mit der teilweise unzureichenden Qualität der Spitaldaten zusammen, die es mit Unterstützung der SwissDRG AG zu verbessern gilt. Für TARPSY Version 2.0 empfiehlt die FMH der SwissDRG AG, die Zusammensetzung der Nebendiagnosenlisten, die Auswirkung von multiplen Nebendiagnosen sowie eine zusätzliche Aufsplittung der Alterskategorien datengestützt zu überprüfen. Es handelt sich bei TARPSY um eine grundlegend neue Tarifstruktur. Umso wichtiger ist es, dass die Tarifpartner für die Einführungsphase Übergangsregelungen vereinbaren und die SwissDRG AG TARPSY jährlich weiterentwickelt.

Literatur

1 HoNOS: Health of the Nation Outcome Scales, Fremdbewertungsinstrument für Erwachsene.

2 Eine hohe Symptomintensität bedeutet hier, dass ein Fall bei mindestens einer der drei HoNOS-Dimensionen einen Wert von 3 oder 4 aufweist. Nur dann kann er eine höher bewertete PCG erreichen.

3 Die Zürcher Hochschule für angewandte Wissenschaften (ZHAW) verwies auf die mangelhafte Datenqualität der Nebendiagnosen des Datenjahrs 2014 und hat deshalb von einem Einbezug in die Vorgängerversion TARPSY 0.3 abgeraten, vgl. ZHAW, Schlussbericht Entwicklung TARPSY 0.3 vom 26.2.2016, Seite 27 f. sowie Seite 126. Die Datenqualität der Nebendiagnosen des Datenjahrs 2015 wird von der SwissDRG AG nicht näher beschrieben.

4 Der Katalog der Zusatzentgelte ist unter www.swissdrg.org unter der jeweils aktuellen SwissDRG-Version im Fallpauschalenkatalog zu finden.

5 Zürcher Hochschule für angewandte Wissenschaften (ZHAW), Schlussbericht Entwicklung TARPSY 0.3 vom 26.2.2016, Seite 123132. 